\title{
Multi-wavelength analysis of the dust emission in the Small Magellanic Cloud ${ }^{\star}$
}

\author{
C. Bot ${ }^{1,2}$, F. Boulanger ${ }^{2}$, G. Lagache ${ }^{2}$, L. Cambrésy ${ }^{1}$, and D. Egret ${ }^{3}$ \\ 1 UMR 7550, Centre de Données Astronomiques de Strasbourg (CDS), Université Louis Pasteur, 67000 Strasbourg, France \\ e-mail: bot@astro.u-strasbg.fr \\ 2 Institut d'Astrophysique Spatiale, Université Paris-Sud, 91405 Orsay, France \\ 3 UMS 2201, Observatoire de Paris, 75014 Paris, France
}

Received 19 December 2003 / Accepted 28 April 2004

\begin{abstract}
We present an analysis of dust grain emission in the diffuse interstellar medium of the Small Magellanic Cloud (SMC). This study is motivated by the availability of $170 \mu \mathrm{m}$ ISOPHOT data covering a large part of the SMC, with a resolution making it possible to disentangle the diffuse medium from the star forming regions. After data reduction and subtraction of Galactic foreground emission, we used the ISOPHOT data together with HiRes IRAS data and ATCA/Parkes combined H I column density maps to determine dust properties for the diffuse medium. We found a far infrared emissivity per hydrogen atom 30 times lower than the Solar neighborhood value. The modeling of the spectral energy distribution of the dust, taking into account the enhanced interstellar radiation field, gives a similar conclusion for the smallest grains (PAHs and very small grains) emitting at shorter wavelength. Assuming Galactic dust composition in the SMC, this result implies a difference in the gas-to-dust ratio (GDR) 3 times larger than the difference in metallicity. This low depletion of heavy elements in dust could be specific of the diffuse ISM and not apply to all the SMC dust if it results from efficient destruction of dust by supernovae explosions.
\end{abstract}

Key words. ISM: dust, extinction - galaxies: Magellanic Clouds - ISM: abundances - ISM: evolution - galaxies: ISM

\section{Introduction}

The Small Magellanic Cloud (SMC) is a dwarf irregular galaxy at a distance of approximately $60 \mathrm{kpc}$ (Westerlund 1991). Its relative proximity makes it possible to have a global view of the galaxy, as well as a sufficiently good resolution to disentangle the diffuse medium from the star forming regions and molecular clouds. The SMC is an ideal target to study the lowmetallicity diffuse medium present not only in dwarf galaxies but also in the external parts of large spirals.

The SMC contains several $\mathrm{H}_{\text {II }}$ regions and molecular clouds (Rubio et al. 1993a). It has a high interstellar radiation field (ISRF), between 4 and 10 times that of the Solar neighborhood (Lequeux 1979; Vangioni-Flam et al. 1980; Azzopardi et al. 1988). It is extremely rich in neutral atomic gas (StaveleySmith et al. 1998) and has a low heavy element abundance (Dufour 1984; Sauvage \& Vigroux 1991). All of these characteristics are indicative of a "young" galaxy, actively forming stars, which might in some sense be considered as a local model of distant galaxies at the beginning of their evolution.

^ Based on observations with ISO, an ESA project with instruments funded by ESA Member States (especially the PI countries: France, Germany, The Netherlands and the UK) and with the participation of ISAS and NASA.
Dust grains are composed mainly of heavy atoms (e.g. carbon, silicium, oxygen, ...). In a low-metallicity environment, such elements are lacking. How does this deficiency act on the dust grain abundances? The gas-to-dust ratio (GDR) is often assumed to be proportional to the metallicity (i.e. there is a fixed fraction of heavy elements in dust). Based on a model of the evolution of elemental abundances in gas and dust, Dwek (1998) concludes that this proportionality applies across the disk of the Galaxy. However, observations in dwarf irregular and blue compact dwarf galaxies show a large scatter in the GDR versus metallicity relation. This indicates that a simple scaling might be a too simplistic description of the phenomenon (Issa et al. 1990; Lisenfeld \& Ferrara 1997, 1998). The GDR is expected to vary among ISM components because destruction of dust by shock waves is more efficient in the diffuse gas while accretion of heavy elements on dust dominates in molecular clouds. The integrated GDR is thus expected to depend on the relative fraction of matter in diffuse/dense components and on the supernova rate and thereby on the star formation history (Hirashita 1999).

The goal of our study is to characterize the dust properties in the diffuse medium of the SMC, and in particular the GDR. Comparing the GDR and the dust spectral energy distribution for the SMC and the Galaxy, we quantify the effect of the 
difference in metallicity on dust abundance and size distribution (e.g. the relative amounts of PAHs, very small grains and big grains).

Since the gas and the dust masses are closely mixed, where the radiation is homogeneous the dust emission is expected to correlate with the distribution of the gas. In the diffuse medium gas is mainly composed of neutral hydrogen, and the GDR can be derived from the comparison of the dust infrared emission with the H i column density, provided that the dust temperature is known. This is not the case in star-forming regions, where the infrared emission from dust in $\mathrm{H}_{\text {II }}$ regions and molecular clouds closely associated with luminous stars can become dominant. Several authors have computed GDRs, comparing the IRAS infrared emission with the $\mathrm{H}_{\text {I }}$ column density, taking the $100 \mu \mathrm{m} / 60 \mu \mathrm{m}$ intensity ratio to derive the dust temperature (Schwering \& Israel 1989; Sauvage et al. 1990; Stanimirovic et al. 2000), but this ignores the contribution from small grains at $60 \mu \mathrm{m}$. An additional difficulty comes from the variations in the intensity of the radiation field inside a beam, which casts doubt on any GDR estimate from studies where the angular resolution is too coarse to separate star forming regions from the diffuse emission (Li \& Draine 2002; Aguirre et al. 2003)

The availability of $170 \mu \mathrm{m}$ ISOPHOT observations enables us to take a new view on dust properties of the SMC. Independently of our work, Wilke et al. (2003) also reduced and analyzed these data but with a distinct focus on point sources. In a second paper (Wilke et al. 2004), these authors address the same question as we do but they investigate the main body (bar) of the SMC without discarding the star forming regions.

After data reduction and validation (Sects. 2 and 3), the $170 \mu \mathrm{m}$ map is compared to additional observations (Sect. 4), in particular the IRAS High Resolution map (HiRes) at $100 \mu \mathrm{m}$. Having obtained the $170 / 100 \mu \mathrm{m}$ emission ratio, in Sect. 5.1 we derive a reference temperature for the big grains in the diffuse medium of the SMC. An H I column density map from ATCA/Parkes combined data (Stanimirovic et al. 1999) is used to study the dust to gas correlation, allowing us to estimate the emissivity of the dust per hydrogen atom for the diffuse medium (Sect. 5.2). Finally, the spectral energy distribution of the dust grains in the diffuse medium of the SMC is fitted with the Desert et al. (1990) model to quantify the abundances of the different grain components (Sect. 5.4). The results are discussed in Sect. 6; Sect. 7 presents our conclusions.

\section{The data}

This study relies on archive data. Some were ready-made such as the IRAS maps at $12,25,60$, and $100 \mu \mathrm{m}$, or the ATCA/Parkes combined data of the $\mathrm{HI}_{\mathrm{I}}$ line at $21 \mathrm{~cm}$. The ISOPHOT data were imported from the ISO Data Archive ${ }^{1}$. But the quality of the ISOPHOT map at $170 \mu \mathrm{m}$ in the ISO archive was not sufficient for this study, so we retrieved, reduced and calibrated the data. We first present the ISOPHOT data and the reduction. Then we describe the ancillary data that were used.

\footnotetext{
${ }^{1}$ http://iso.vilspa.esa.es/ida/index.html
}

\subsection{The ISOPHOT data}

The Small Magellanic Cloud was almost fully covered by 10 observations with the ISOPHOT instrument (Lemke et al. 1996) on board the ISO satellite (Kessler et al. 1996; Laureijs et al. 2003). The observations were made using the P22 AOT (Astronomical Observation Template), i.e. a raster map on a two-dimensional regular grid, with the C200 detector $(2 \times 2$ pixels $)$ at an effective wavelength of $170 \mu \mathrm{m}$. The pixel field of view is $1.5^{\prime}$ and the raster pointing spacings are $180^{\prime \prime}$ in both in-scan and cross-scan directions. The ISOPHOT point spread function can be approximated by a 2D Gaussian with $90^{\prime \prime}$ full width half maximum (FWHM) in both directions and is supposed to be constant across the map.

We reduced the data with PIA $^{2}$ (ISOPHOT Interactive Analysis) V10.0 (Gabriel et al. 1997, 1998), except for the flatfield correction. In the following, we describe the specific treatments applied. Some observations showed specific problems in the raw data (e.g. a pixel went into erratic state or a severe glitch affected a pixel until the end of the observation). We chose to remove the data for these pixels in the corresponding observations. If this removal is not done, the reduction processes will interpret these erratic fluxes as flat-fielding. This distorts the photometry of the other pixels for the whole observation.

To deal with the discarded pixels, we chose to perform the flat-field correction without the PIA tool as in Lagache \& Dole (2001). The flat-field correction is on average $1.01 \pm 0.05$, $0.93 \pm 0.03,1.17 \pm 0.09$ and $0.92 \pm 0.03$ for pixel $1,2,3$ and 4 respectively. Reproducible flat-field correction values for the ISOPHOT C_200 array have been published by Lagache \& Dole (2001) from the careful reduction of 3 deep fields (FIRBACK). We do not observe the same average flat field correction factor and we find a higher dispersion from one observation to another. This difference may be because the responsivity of ISOPHOT C_200 pixels changes with the observed intensity, so that the flat-field correction computed with low fluxes is no longer valid for the brightest pixels (Pagani et al. 2003). This might affect the $170 \mu \mathrm{m}$ flux determination for bright sources. The reproducible behavior of pixels observed by Lagache $\&$ Dole (2001) is then due to the relative flatness of deep fields.

The different observations have been done at different times, therefore the "absolute calibration" performed using the FCS measurement can be slightly different from one raster to another. This difference is observed as discontinuities between maps in the mosaic. To correct for these small absolute calibration differences (for a border between two maps, the mean difference in brightness observed is about $13 \%$ ), we applied a "general flat-field" correction as in Lagache \& Dole (2001).

After concatenation of the timelines, the raster is projected on the sky using the coordinates of each individual pixel, with a pixel size for the map of $10^{\prime \prime}$ to match the IRAS HiRes

2 The ISOPHOT data presented in this paper were reduced using PIA, which is a joint development by the ESA Astrophysics Division and the ISOPHOT Consortium with the collaboration of the Infrared Processing and Analysis Center (IPAC). Contributing ISOPHOT Consortium institutes are DIAS, RAL, AIP, MPIK, and MPIA. 


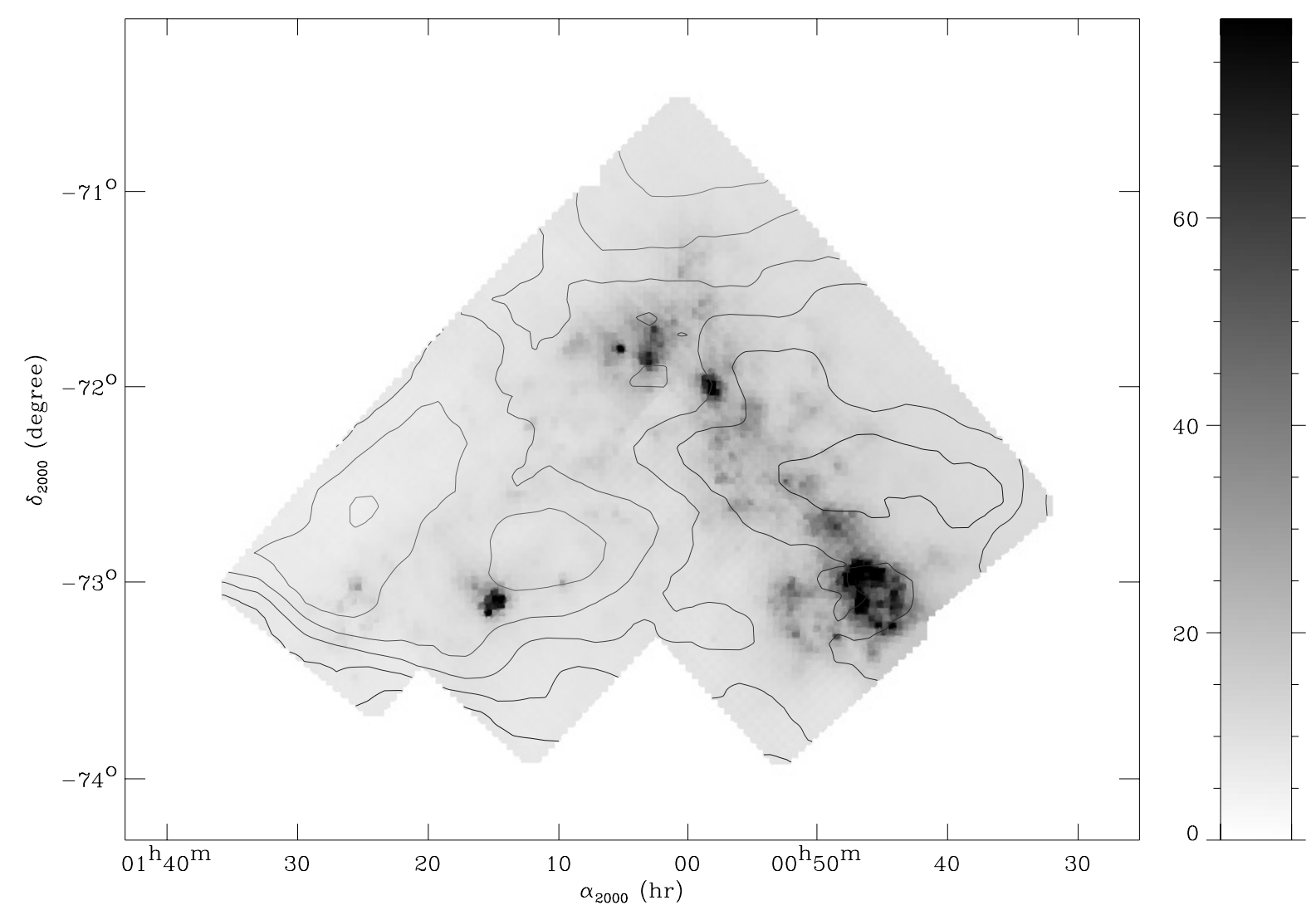

Fig. 1. The ISOPHOT map at $170 \mu \mathrm{m}$ (in MJy/sr) after reduction and bilinear interpolation to fill the holes left by the mapping pattern. The flat-field has been efficiently corrected for the diffuse part of the map, but one can still see low level defects in the brightest parts. Contours of the $\mathrm{H}_{\mathrm{I}}$ Galactic foreground are overplotted at column densities of 2.6, 2.8, 3., 3.2, 3.4 and $3.6 \times 10^{20} \mathrm{H}_{\text {atom cm}}{ }^{-2}$ (from light grey to black).

gridding. Because the raster step size of each observation was a full detector size, and the spacecraft $Y$-axis was not parallel to right ascensions or declinations, we observe gaps between the individual array pointings. The final map is presented in Fig. 1 after a bilinear interpolation to reconstruct the signal in the gaps.

\subsection{Ancillary data}

\subsubsection{IRAS HiRes data}

As a complement to the ISOPHOT data at $170 \mu \mathrm{m}$, we used IRAS high resolution (HiRes) data at 12, 25, 60 and $100 \mu \mathrm{m}$. Maps of $2^{\circ} \times 2^{\circ}$ centered at $00^{\mathrm{h}} 57^{\mathrm{m}} 29.12^{\mathrm{s}}-72^{\circ} 32^{\prime} 29.2^{\prime \prime}$ in J2000 were obtained. The pixel size is $10^{\prime \prime}$. The HiRes point spread function (PSF) varies spatially in each map. A Gaussian approximation of the PSF is given with the HiRes maps and is defined by two spatially dependent $F W H M$ s and a rotation angle. For more information on HiRes, please refer to Aumann et al. (1990) and Bontekoe et al. (1994). Since we are searching for the properties of extended structures, the HiRes IRAS maps were brought to the DC-calibration (Wheelock et al. 1994). This DC calibration is only valid for spatial scales greater than $40^{\prime}$. As the SMC exhibits structure on various spatial scales, the DC calibration introduces some limitations that will be discussed in Sect. 3.2.

\subsubsection{ATCA/Parkes combined SMC H। data}

To study the correlation between gas and dust in the SMC, we needed a $\mathrm{H}_{\mathrm{I}}$ column density map to compare with the ISOPHOT map, in order to compute the abundances of dust grains relative to hydrogen.

Stanimirovic et al. (1999) have combined Parkes telescope observations of neutral hydrogen in the SMC with the Australia Telescope Compact Array (ATCA) aperture synthesis mosaic to obtain a set of images sensitive to all angular spatial scales between $98^{\prime \prime}$ and $4^{\circ}$ from 90 to $215 \mathrm{~km} \mathrm{~s}^{-1}$. The spatial resolution obtained is $98^{\prime \prime}$ and the velocity resolution is $1.65 \mathrm{~km} \mathrm{~s}^{-1}$. We used a column density map of the SMC obtained by integrating over the whole velocity range and assuming that the neutral hydrogen is optically thin ${ }^{3}$.

\subsubsection{Parkes $\mathrm{H}$ I data for the Galactic foreground}

A Galactic filament exists in front of the SMC. Even though it is very smooth, large (see contours of Fig. 1) and uniform (column densities between $2.5 \times 10^{20}$ and $4 \times 10^{20}$ atom $\mathrm{cm}^{-2}$ ), it can bias studies. The far-infrared fluxes observed in either the ISOPHOT or the IRAS HiRes maps represent the emission from the SMC dust, but also from this Galactic foreground. However, this Galactic component of the emission is not

\footnotetext{
3 The column density map and the data cube are available at http://www.atnf.csiro.au/research/smc_h1
} 


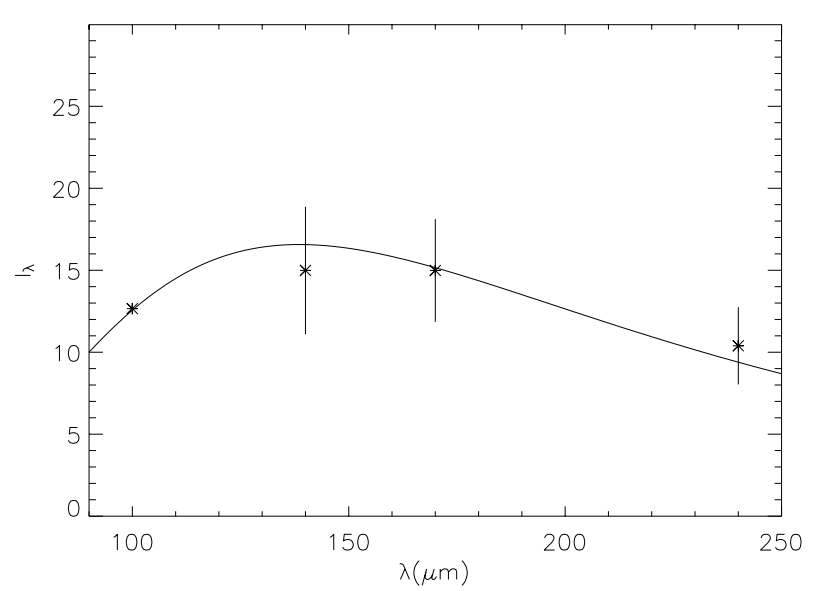

Fig. 2. Comparison of the DIRBE-convolved ISOPHOT fluxes with the DIRBE fluxes (in MJy/sr), after integration on the whole SMC. The modified black body traced was fit to the DIRBE values only and corresponds to a temperature of $20.6 \mathrm{~K}$ and an emissivity of $\epsilon_{\mathrm{H}}(250 \mu \mathrm{m})=3 \times 10^{-28} \mathrm{~cm}^{2}$ for $10^{20}$ hydrogen atoms $\mathrm{cm}^{-2}$. We see that the ISOPHOT value at $170 \mu \mathrm{m}$ is consistent with the DIRBE photometry.

observed in the ATCA/Parkes data, due to a velocity cut and spatial filtering. To study the SMC dust properties, it was thus necessary to remove this foreground component (see Sect. 3.3). In this step, we used a Galactic H I column density map constructed from data of the Parkes narrow-band H i survey of the tidal arms of the Magellanic system (Brüns et al. 2000). The column density map of the $\mathrm{H}_{\text {I }}$ Galactic foreground was obtained by integrating the emission from -60 to $+50 \mathrm{~km} \mathrm{~s}^{-1}$, outside the SMC velocities. The resolution is $15^{\prime}$. Due to side-lobe effects, the map obtained can overestimate the column density by 10 to $20 \%$.

\section{Photometric consistency}

DIRBE provided infrared absolute sky brightness maps in 10 bands in the wavelength range 1.25 to $240 \mu \mathrm{m}$. This absolute photometry is used to check ISOPHOT mosaic and IRAS fluxes.

\subsection{ISOPHOT data}

We checked if the ISOPHOT $170 \mu \mathrm{m}$ flux is consistent with the spectral energy distributions obtained from the 100, 140 and $240 \mu \mathrm{m}$ DIRBE fluxes in the SMC. To do this we convolved the ISOPHOT map with the DIRBE beam. This gives 18 ISOPHOT fluxes in the SMC as if they were observed by DIRBE, and we compare ISOPHOT and DIRBE fluxes by plotting the spectral energy distribution and fitting it with a modified black body with a spectral index of 2 on the DIRBE values. Figure 2 shows that the DIRBE-convolved ISOPHOT flux integrated over the 18 positions observed by DIRBE, is consistent within the errors bars with the best modified blackbody fit (obtained for a temperature of $20.6 \mathrm{~K}$, an emissivity of $\epsilon_{\mathrm{H}}(250 \mu \mathrm{m})=3 \times 10^{-28} \mathrm{~cm}^{2}$ for $10^{20}$ hydrogen atoms $\left.\mathrm{cm}^{-2}\right)$. Please note that these values correspond to an average over the whole galaxy and were obtained only for calibration purposes.
We have also plotted the individual fit values at $170 \mu \mathrm{m}$ versus the corresponding 18 ISOPHOT values. This plot shows a significant scatter but is consistent with a linear correlation with unity slope and zero offset.

A comparison with the IRAS high resolution data was also performed to check possible photometric errors introduced by the interpolation process. The correlation between IRAS and ISOPHOT data does not change when we restrict the comparison to the pixels measured by ISOPHOT rather than the full interpolated map. The interpolation required to fill the holes in the measurements does not seem to bias the image comparisons.

\subsection{IRAS data}

The IRAS HiRes maps have the same photometric calibration as the IRAS ISSA maps. The IRAS detector had a dwell-time dependent responsivity change: the gain changes as a function of source size. This effect is known as the "AC/DC effect". A first set of corrections was taken into account in the IRAS data products. These corrections were later found to be insufficient. On the basis of a comparison of the IRAS and DIRBE sky brightness for extended emission, Wheelock et al. (1994) recommended applying additional correction factors of $0.88,1.01$, 0.82 and 0.74 at $12,25,60$ and $100 \mu \mathrm{m}$ respectively.

However, these correction factors are only valid for spatial scales greater than $40^{\prime}$. In the SMC structures on various spatial scales are present. Stanimirovic et al. (2000) have also compared DIRBE and IRAS data by integrating fluxes over $6.25^{\circ 2}$ in the SMC. They found that integrated flux densities measured by DIRBE for 60 and $100 \mu \mathrm{m}$ are higher than IRAS fluxes by about 10 to $20 \%$, which is the opposite correction to that found by Wheelock et al. (1994).

We have compared the IRAS (convolved to the DIRBE resolution) and the DIRBE $100 \mu \mathrm{m}$ maps on a $10^{\circ} \times 10^{\circ}$ scale around the SMC to investigate this discrepancy. We see that the Wheelock et al. (1994) correction factor of 0.74 is only truly valid outside the galaxy and increases in the SMC up to a maximum value located in the north of the optical bar (N66 region). For the diffuse medium of the SMC, the Wheelock et al. (1994) correction factor is sufficient. After correction, we estimate that the $100 \mu \mathrm{m}$ IRAS brightness for the diffuse medium is underestimated by less than $15 \%$.

\subsection{Galactic foreground removal}

Unlike the ATCA/Parkes Hi map, the far-infrared emission maps (ISOPHOT and IRAS) trace both the dust emission in the SMC and the dust in our Galaxy. To disentangle these two components in the far-infrared emission, we used the column density map of the H I Galactic foreground presented in Sect. 2.2.3.

Dust grains absorb energy from the UV/optical radiation field which they re-emit in the infrared as thermal emission. Large grains are at a fixed temperature and their emitted intensity can be expressed as:

$I_{v}=N_{\mathrm{H}} \epsilon_{\mathrm{H}}(v) B_{v}\left(T_{\text {dust }}\right)$ 

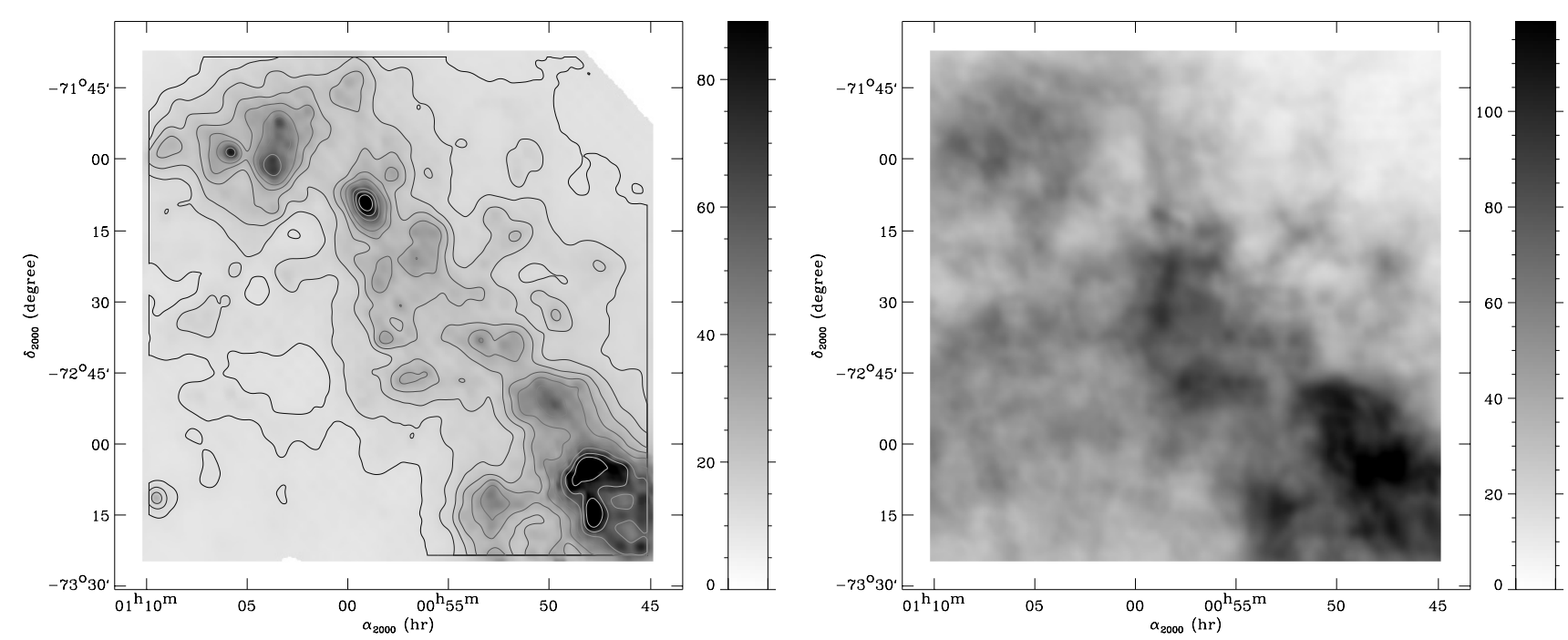

Fig. 3. ISOPHOT map (left panel, in MJy/sr) and ATCA/Parkes $\mathrm{H}_{\text {I }}$ column density map (right panel, in $10^{20}$ hydrogen atom/ $\mathrm{cm}^{2}$ ) after projection on the IRAS HiRes grid, and at the same resolutions. For the ISOPHOT map, logarithmic contours are overplotted at fluxes 11.2, 14.1, $17.8,22.4,28.2,35.5,44.7,56.2,70.8$ and $89.1 \mathrm{MJy} / \mathrm{sr}$.

where $N_{\mathrm{H}}$ is the column density of hydrogen and $\epsilon_{\mathrm{H}}(v)$ is the emissivity of grains per hydrogen atom. We assume that the emissivity of dust grains in the far-infrared follows a power law:

$\epsilon_{\mathrm{H}}(v)=\epsilon_{\mathrm{H}}\left(v_{0}\right)\left(\frac{v}{v_{0}}\right)^{\beta}$

and we take $\beta=2$ (as found for Galactic emission (Lagache et al. 1998, 1999) for wavelengths between $100 \mu \mathrm{m}$ and $500 \mu \mathrm{m})$.

Using a temperature of $17.5 \mathrm{~K}$ and an emissivity per hydrogen atom of $10^{-25} \mathrm{~cm}^{2}$ (Boulanger et al. 1996a) ${ }^{4}$ for the Galactic dust, we computed the Galactic thermal emission and removed this foreground emission from the IRAS and ISOPHOT maps to obtain the emission of the SMC dust only. At 12,25 and $60 \mu \mathrm{m}$, we used the $I_{12} / I_{100}, I_{25} / I_{100}$ and $I_{60} / I_{100}$ ratios computed from IRAS maps outside the SMC, together with the computed foreground emission map at $100 \mu \mathrm{m}$ to remove the Galactic foreground emission at these wavelengths. However, this foreground emission remains in the data noise at 12,25 and $60 \mu \mathrm{m}$.

\section{Spatial image comparison}

\subsection{Projection and resolution}

The data set presented in Sect. 2 is composed of maps with different fields of view and different resolutions. We projected all of them onto a common grid: that of IRAS HiRes maps $\left(2^{\circ} \times 2^{\circ}\right.$ field of view centered at $00^{\mathrm{h}} 57^{\mathrm{m}} 29.12^{\mathrm{s}}-72^{\circ} 32^{\prime} 29.2^{\prime \prime}$ (J2000) with $10^{\prime \prime}$ pixel size). These maps were inter-compared by pairs.

To compare the ISOPHOT map with one of the HiRes maps, the projected and re-sampled ISOPHOT map was convolved with a spatially varying kernel in order to be at the

\footnotetext{
${ }^{4}$ We checked that the observed IRAS and DIRBE far-infrared ratios around the SMC are consistent with these values.
}

HiRes resolution when the point spread function of ISOPHOT was smaller than that of HiRes (Kerton \& Martin 2001). The HiRes map was also convolved with a spatially varying kernel to match the ISOPHOT resolution when the HiRes beam was smaller than the ISOPHOT one.

To compare the HI column density map with the ISOPHOT map, no convolution was necessary as long as their resolutions were comparable.

Figure 3 shows two of the transformed maps: the ISOPHOT map degraded to the IRAS $100 \mu \mathrm{m}$ resolution and the projected ATCA/Parkes map.

\subsection{Colors from scatter plots}

To characterize the SED of the diffuse medium (i.e. the extended region where the infrared emission-H I column density correlation is homogenous), we developed a comparison method that is not biased by point sources and is also not affected by offsets in the data calibration.

We work with two maps (obtained after the treatment presented in Sect. 4). The general correlation between the two maps is first quantified by fitting a straight line to the scatter plot of the pixel-to-pixel comparison. We deduce a first value for the ratio and an error $\sigma$ given by the dispersion in the correlation, which enables us to filter out localized sources (i.e. star-forming regions) by removing pixels that are $3 \sigma$ above the correlation (there are no points below $-3 \sigma$ ).

From the "filtered" maps, we compute a ratio map by linearly fitting the pixel correlation in the scatter plot for a sliding box of $50 \times 50$ pixels. This corresponds to a $5.5 \times 5.5$ beams box which is a minimum size to have independent data points. The size of the box could not be greater, or we would not trace the local variations. In some cases there are too few points inside a box (too many points above $3 \sigma$ ) to do a meaningful fit. We assign no value to these positions. We also skip positions where the correlation coefficient is too low. Adjusting a Gaussian to 

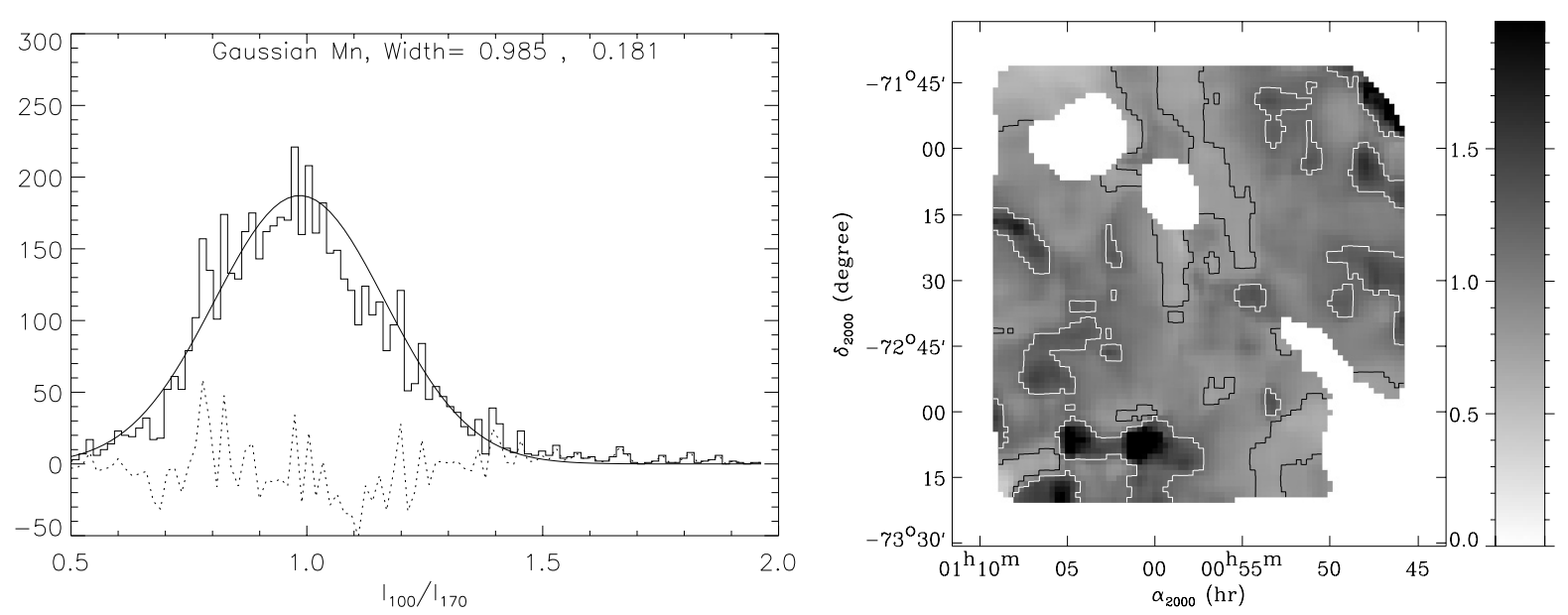

Fig. 4. Histogram and spatial distribution of the 100-170 $\mu \mathrm{m}$ colors. For the histogram, the Gaussian fit and the residuals are presented as continuous and dotted lines respectively. For the map of the 100-170 $\mu \mathrm{m}$ colors, contours correspond to the 0.8 (black) and 1.2 (white) levels (one sigma limits of the Gaussian fit). It should be noticed that the noise in the color map is not homogeneous.

the histogram of the ratio values gives us a reference ratio and the associated dispersion. This dispersion contains photometric and fitting errors but also a true scatter in the ratio. The two phenomena cannot be easily disentangled.

The spatial distribution and the histogram with the Gaussian fit obtained for the $I_{100 \mu \mathrm{m}} / I_{170 \mu \mathrm{m}}$ ratio are presented in Fig. 4 as an example. We see that the comparison method avoids large areas in the main body (bar) of the SMC. These regions correspond to highly structured star-forming sites. In these regions, either the filtering technique has left too few independent positions in one box to compute a significant correlation or no clear correlation is observed.

\section{Results}

\subsection{Infrared colors}

The method presented in Sect. 4.2 was applied to the IRAS HiRes and ISOPHOT maps to compute the $I_{100 \mu \mathrm{m}} / I_{170 \mu \mathrm{m}}, \quad I_{60 \mu \mathrm{m}} / I_{170 \mu \mathrm{m}}, \quad I_{25} \mu \mathrm{m} / I_{170 \mu \mathrm{m}}, \quad I_{12} \mu \mathrm{m} / I_{170 \mu \mathrm{m}}$ ratios. Adjusting a Gaussian to the histograms gives us reference ratios and their associated dispersions:

$$
\begin{aligned}
& \frac{I_{100 \mu \mathrm{m}}}{I_{170 \mu \mathrm{m}}}=1.0 \pm 0.2 \\
& \frac{I_{60 \mu \mathrm{m}}}{I_{170 \mu \mathrm{m}}}=0.5 \pm 0.1 \\
& \frac{I_{25 \mu \mathrm{m}}}{I_{170 \mu \mathrm{m}}}=0.02 \pm 0.01 \\
& \frac{I_{12 \mu \mathrm{m}}}{I_{170 \mu \mathrm{m}}}=0.011 \pm 0.006 .
\end{aligned}
$$

Using Eqs. (1) and (2) we can derive from the $\frac{I_{100 \mu \mathrm{m}}}{I_{170} \mu \mathrm{m}}$ ratio map the big grain equilibrium temperature distribution in the diffuse medium in the Small Magellanic Cloud. This leads to a reference temperature $T_{\text {dust }}=22 \pm 2 \mathrm{~K}$. Note that this temperature is different from the one that would be inferred from the ratios of the mean $I_{100 \mu \mathrm{m}}$ and $I_{170 \mu \mathrm{m}}$ fluxes. Wilke et al. (2004) found a mean temperature for the main body of the SMC of $20.9 \pm 1.9 \mathrm{~K}$, which is lower than the temperature we find for the diffuse medium. Their mean value falls within our range of temperatures, which is expected because the regions we study overlap. The difference in mean values could be due to the presence of cold atomic and molecular clouds in the main body of the SMC. Our studies seem to be consistent with each other and are more complementary than comparable.

The large dust grain temperature is related to the radiation field intensity and will be discussed in Sect. 6.

\subsection{Gas to dust correlation}

From the ISOPHOT and the ATCA/Parkes maps, using the method described Sect. 4.2, we found the distribution presented in Fig. 5. This distribution exhibits a main peak and a distribution tail. The peak is characterized by:

$$
\frac{I_{170 \mu \mathrm{m}}}{N_{\mathrm{HI}}}=0.17 \times 10^{-20} \pm 0.06 \times 10^{-20} \mathrm{MJy} \mathrm{sr}^{-1} \mathrm{~cm}^{2} \text {. }
$$

Since the regions used to define the ratios are not exactly the same, the determination of the characteristics of the diffuse medium could be biased. We checked that this is not the case. If we use the region where $I_{170 \mu \mathrm{m}} / N_{\mathrm{HI}}$ is computed to determine the colors, we find that the derived quantities are not affected by more than $15 \%$. We see that the characteristics of the diffuse medium are not biased by the definition of the limits of the studied region. Comparing the region defined by Eq. (7) with the $\mathrm{H} \alpha$ distribution, we see in Fig. 6 that the regions corresponding to the tail in the $I_{170 \mu \mathrm{m}} / N_{\mathrm{HI}}$ (located mainly in the main body) are regions where the diffuse $\mathrm{H} \alpha$ emission is important. Comparing the maps in Figs. 4 and 6, we also see that the regions where no ratios can be computed correspond to important star-forming sites.

Assuming that all the dust is in the HI, we use the $I_{170 \mu \mathrm{m}} / N_{\mathrm{HI}}$ ratio map and the temperature map computed above to obtain a histogram of the emissivity per hydrogen atom of 


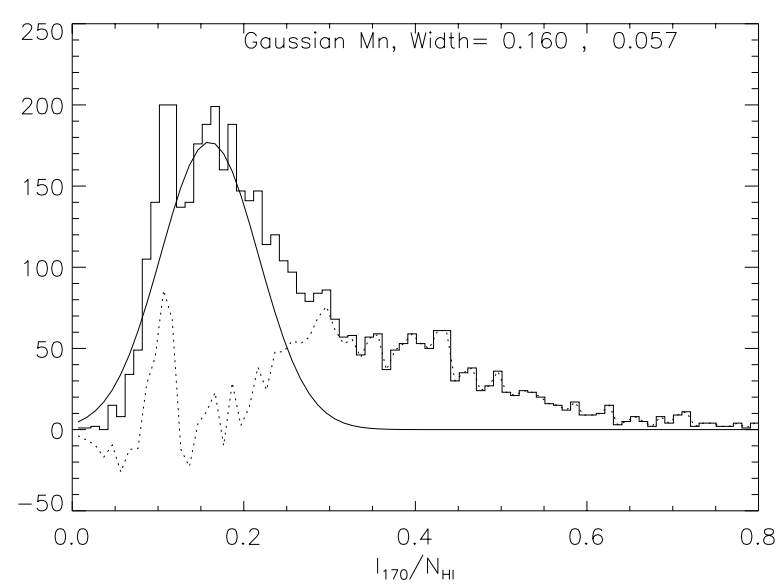

Fig. 5. Histogram of the 170 micron flux to $\mathrm{H}_{\mathrm{I}}$ column density ratio (in $\mathrm{MJy} \mathrm{cm}^{2} / \mathrm{sr}$ ). The Gaussian fit and the residuals are presented as continuous and dotted lines respectively.

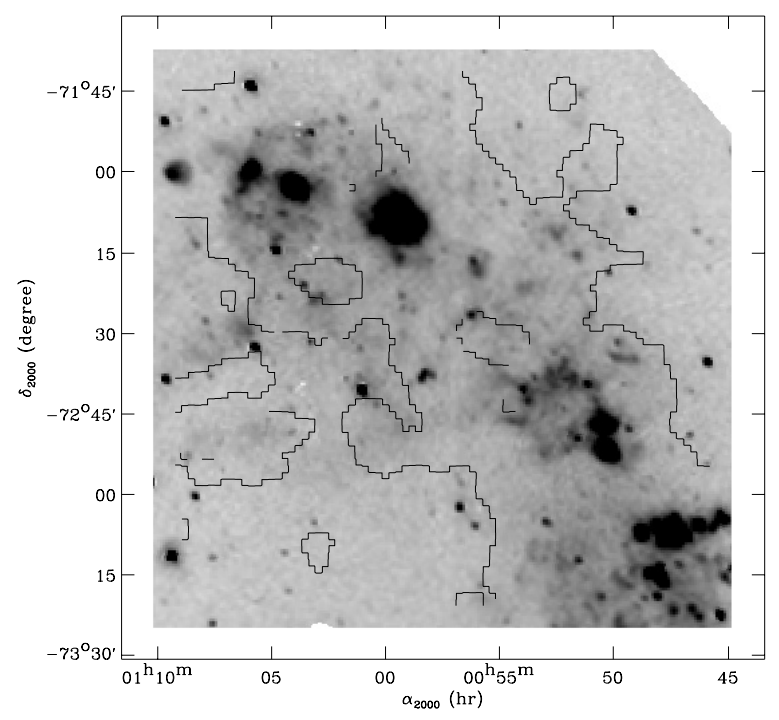

Fig. 6. Uncalibrated $\mathrm{H} \alpha$ map. As an illustration, the contour line corresponds to $I_{170 \mu \mathrm{m}} / N_{\mathrm{HI}}=0.23 \mathrm{MJy} \mathrm{cm}^{2} / \mathrm{sr}$ (see Eq. (7)) as in Fig. 7.

the dust grains in the diffuse medium. We find a reference emissivity at $170 \mu \mathrm{m}$ of:

$\epsilon_{\mathrm{H}}(\lambda)=7.3 \times 10^{-27} \pm 3.2 \times 10^{-27} \mathrm{~cm}^{2}$.

The error takes into account uncertainties in the temperature and $I_{170 \mu \mathrm{m}} / N_{\mathrm{HI}}$. If the emissivity follows a power law with a spectral index of 2, the emissivity per $\mathrm{HI}_{\mathrm{I}}$ atom in the SMC at $250 \mu \mathrm{m}$ is $\epsilon_{\mathrm{H}}(\lambda)=3.4 \times 10^{-27} \pm 1.5 \times 10^{-27}(\lambda / 250 \mu \mathrm{m})^{-2} \mathrm{~cm}^{2}$. This corresponds to $\kappa_{v}=0.21(\lambda / 250 \mu \mathrm{m})^{-2} \mathrm{~cm}^{2} \mathrm{~g}^{-1}$. Assuming similar dust optical properties in the SMC and the Galaxy, the emissivity is related to the GDR and the ratio between hydrogen column density and $E(B-V)$ color excess by:

$\frac{\epsilon_{\mathrm{H}}(\mathrm{SMC})}{\epsilon_{\mathrm{H}}(\text { Galaxy })}=\frac{\frac{M_{\text {dust }}}{M_{\text {gas }}}(\mathrm{SMC})}{\frac{M_{\text {dust }}}{M_{\text {gas }}}(\text { Galaxy })}=\frac{\frac{N\left(\mathrm{H}_{\mathrm{I}}\right)+N\left(\mathrm{H}_{2}\right)}{E(B-V)}(\text { Galaxy })}{\frac{N\left(\mathrm{H}_{\mathrm{I}}\right)+N\left(\mathrm{H}_{2}\right)}{E(B-V)}(\mathrm{SMC})}$.

The solar neighborhood value for the emissivity is $10^{-25} \mathrm{~cm}^{2}$ at $250 \mu \mathrm{m}$ (Boulanger et al. 1996a). The inferred SMC GDR

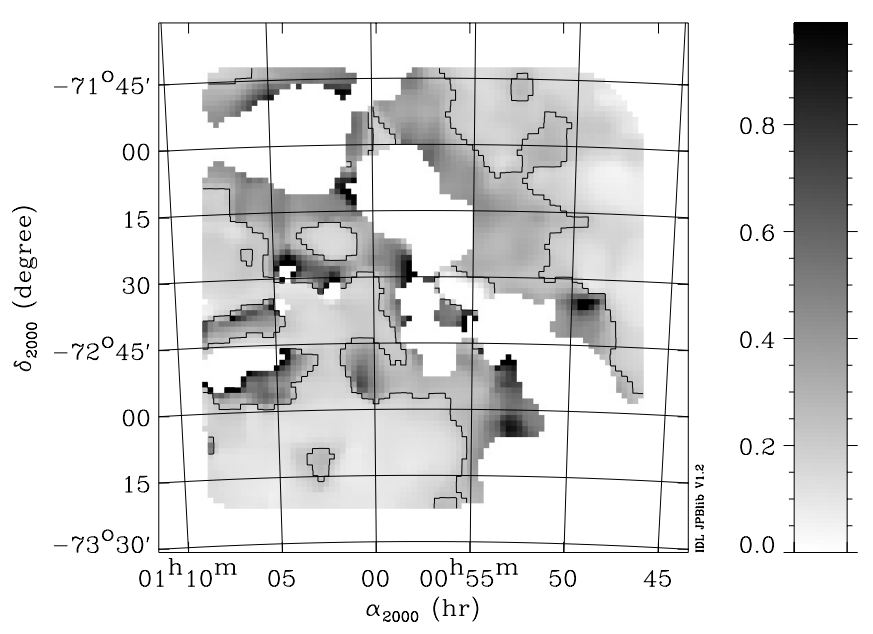

Fig. 7. Spatial distribution of the $I_{170 \mu \mathrm{m}} / N_{\mathrm{HI}}$ ratio (in $\mathrm{MJy} \mathrm{cm}^{2} / \mathrm{sr}$ ). Regions where no ratio is computed (see Sect. 4.2) are left blank. The contour line corresponds to a $I_{170 \mu \mathrm{m}} / N_{\mathrm{HI}}=0.23 \mathrm{MJy} \mathrm{cm}^{2} / \mathrm{sr}$ level. We see that the IR excesses are concentrated near the optical bar.

is thus 30 times greater than in the Galaxy. Stanimirovic et al. (2000) have presented a similar study using the same H I data together with HIRAS data. They came to the same conclusion that the GDR is a factor 30 higher in the SMC than in our Galaxy. However, this agreement might be a coincidence since 1) we did not use the same calibration of the IRAS data; 2) their temperature estimation based on the $60 / 100 \mu \mathrm{m}$ color is higher than ours; 3 ) the GDR they present is averaged over the whole galaxy (including star forming regions); and 4) their GDR is compared to a Galactic GDR of 375 instead of the standard value of $100^{5}$. Using the standard Galactic GDR value, the average SMC GDR found by Stanimirovic et al. (2000) becomes 100 times higher than in the Galaxy.

Our estimate of the SMC's GDR of 30 times higher than in the Galaxy, is significantly larger than the difference in metallicity, which is less than 10 (Dufour 1984). It is twice the observed difference in the $\frac{N\left(\mathrm{H}_{I}\right)+N\left(\mathrm{H}_{2}\right)}{E(B-V)}$ values presented by Tumlinson et al. (2002).

\subsection{Infrared excesses}

A noticeable result of the ISOPHOT and H I comparison is the presence of a tail in the $170 \mu \mathrm{m} / \mathrm{H}$ I histogram (see Fig. 5). This tail corresponds to pixels in the SMC optical bar with excess $170 \mu \mathrm{m}$ emission with respect to $N_{\mathrm{HI}}$ (see Fig. 7). We checked that the mean temperature for this region of excess is the same as that for the diffuse medium. These excesses may be due either to a variation in the dust abundances between the diffuse medium and the bar, or to the presence of cold $\mathrm{H}_{\mathrm{I}}$ or $\mathrm{H}_{2}$ gas. We believe that the excesses are real but it is difficult to infer an amount of cold $\mathrm{HI}$ and molecular gas from it. Firstly, it is difficult to disentangle these two possible contributions. Secondly, in the regions where some of these excesses are due to dust in cold $\mathrm{H}_{\mathrm{I}}$ or $\mathrm{H}_{2}$ we do not expect them to be

\footnotetext{
5 This value can be checked with the interstellar abundance standards taken from Table 2 of Sofia \& Meyer (2001b) and Sofia \& Meyer (2001a).
} 
correlated with $\mathrm{HI}_{\mathrm{I}}$ emission and another method is needed to quantify them. It is thus beyond the scope of this paper to translate these excesses into an estimate of cold gas that could be compared to other studies where this is attempted (Stanimirovic et al. 2000). Finally, we feel that the photometric uncertainties in the ISOPHOT and IRAS data are an additional difficulty, especially near star-forming regions. Further studies with the Spitzer satellite could address this question with better photometry and angular resolution.

\subsection{SMC dust properties in diffuse $\mathrm{HI}$ : Modeling the spectral energy distribution}

The different intensity ratios obtained in Sect. 5.1 enable us to trace the spectral energy distribution of dust in the diffuse medium of the SMC and to compare it with that of the solar neighborhood. The difference in temperature and in abundances is seen in Fig. 9 as a shift along the wavelength and brightness axis respectively. The error bars include true variations in the dust properties across the SMC diffuse interstellar medium which are correlated over all wavelengths.

We used the dust model of Desert et al. (1990) to quantify the grain dust abundances. To model the spectral energy distribution (SED) we need to estimate the interstellar radiation field (ISRF) in the diffuse medium of the SMC. In Sect. 5.1 we found the big dust grain equilibrium temperature to be $22 \mathrm{~K}$. Using the dust thermal equilibrium temperature in the solar neighborhood of $17.5 \mathrm{~K}$ (Boulanger et al. 1996a) as a reference, the dust temperature in the SMC leads to a radiation field $\chi_{\mathrm{SMC}}=T_{\text {dust }}^{4+\beta}(\mathrm{SMC}) / T_{\text {dust }}^{4+\beta}($ Galaxy $)=4$. This value is consistent with the estimate of the ISRF made from massive star counts by Lequeux (1979). Vangioni-Flam et al. (1980) also found the same ratio from UV measurements. We used the Mathis et al. (1983) $10 \mathrm{kpc}$ Galactic ISRF and scaled it by a factor $\chi=4$ for the modeling. We have adjusted only the abundances of PAHs, very small grains (VSG) and big grains. For the other parameters defining the size distribution we have kept the Galactic values. The best fit is presented in Fig. 8; it corresponds to under-anbundant PAHs, very small grains (VSG) and big grains (BG) compared to the Galaxy $(36,23$ and 30 times less respectively). Mass abundances relative to hydrogen, compared with Galactic values (Desert et al. 1990) are summarized in Table 1 .

The modeling confirms the GDR estimated in Sect. 5.2 to be around 30 times greater than in the Galaxy. Within the uncertainties, PAHs and very small grain abundances are also consistent with this factor.

The model is clearly below the $60 \mu \mathrm{m}$ point. This could be due to our crude estimate of the ISRF. We have used a single ISRF while our data analysis shows temperature variations across the SMC diffuse medium. To take the variations in the radiation field into account, we transformed the temperature distribution obtained in Sect. 5.1 into a $\chi_{\mathrm{SMC}}$ distribution. For each value of the distribution we computed a spectral energy distribution (SED) of the dust emission with the Desert et al. (1990) model, using the Mathis $10 \mathrm{kpc}$ ISRF multiplied by the corresponding $\chi$ as an input $(\chi$ is a constant for the whole

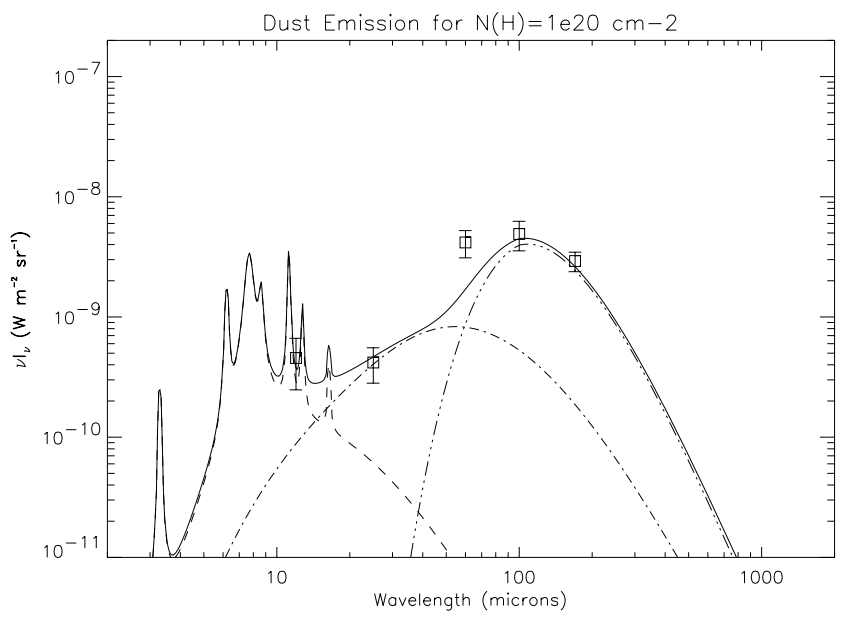

Fig. 8. Best fit for the dust infrared emission spectrum, obtained with the Desert et al. model. The brightness corresponds to an interstellar gas column density of $10^{20} \mathrm{~cm}^{-2}$. The IRAS and ISOPHOT data are represented by squares. The fit obtained with the model (continuous line) corresponds to the emission of three components: PAHs (dashed line), very small grains (mixed line) and big grains (dot-dotdot-dashed line).

Table 1. Mass abundances relative to hydrogen in the SMC and the Galaxy. The grain size distribution is assumed to be the same in both cases.

\begin{tabular}{lll}
\hline \hline Component & $\begin{array}{l}\text { SMC } \\
\frac{m}{m_{\mathrm{H}}}\end{array}$ & $\begin{array}{l}\text { Galaxy } \\
m_{\mathrm{H}}\end{array}$ \\
\hline PAH & $1.2 \times 10^{-5}$ & $4.3 \times 10^{-4}$ \\
VSG & $2.0 \times 10^{-5}$ & $4.7 \times 10^{-4}$ \\
BG & $2.2 \times 10^{-4}$ & $6.4 \times 10^{-3}$ \\
\hline
\end{tabular}

spectrum). We then computed the resulting total SED by adding the individual SEDs with weights according to the $\chi$ distribution shape. The resulting fit is not sufficiently broadened to encompass the $60 \mu \mathrm{m}$ point. In addition, the mass abundances of dust grains have to be reduced by 8,5 and 7 for the PAH, VSG and BG components respectively. Due to the high non-linearity of the Planck curve, even a small broadening of the $\chi$ distribution tends to reduce the abundances of dust grains significantly. These values are thus indicative only but they show that the mass abundances presented in Table 1 are more likely to be upper limits and that the emissivity could be even lower.

It is possible to fit the $60 \mu \mathrm{m}$ value with a single ISRF scaled by $\chi=10$ (see Fig. 10). In this case, the dust grain mass abundances must be reduced by 3,2 and 2 for the PAHs, very small grains and big grains respectively. Such a high ISRF corresponds to a dust temperature of $25.6 \mathrm{~K}$, which is higher than the estimate obtained in Sect. 5.1 but still compatible with the $100 \mu \mathrm{m}$ and the $170 \mu \mathrm{m}$ fluxes. This higher temperature would lead to an emissivity of $2.4 \times 10^{-27} \mathrm{~cm}^{2}$ at $250 \mu \mathrm{m}$, which is even lower than the estimate obtained in Sect. 5.2, and results in a GDR 40 times higher than in the Galaxy. A higher radiation field could thus account for the $60 \mu \mathrm{m}$ excess without contradicting the main conclusion of this study, namely that the GDR 


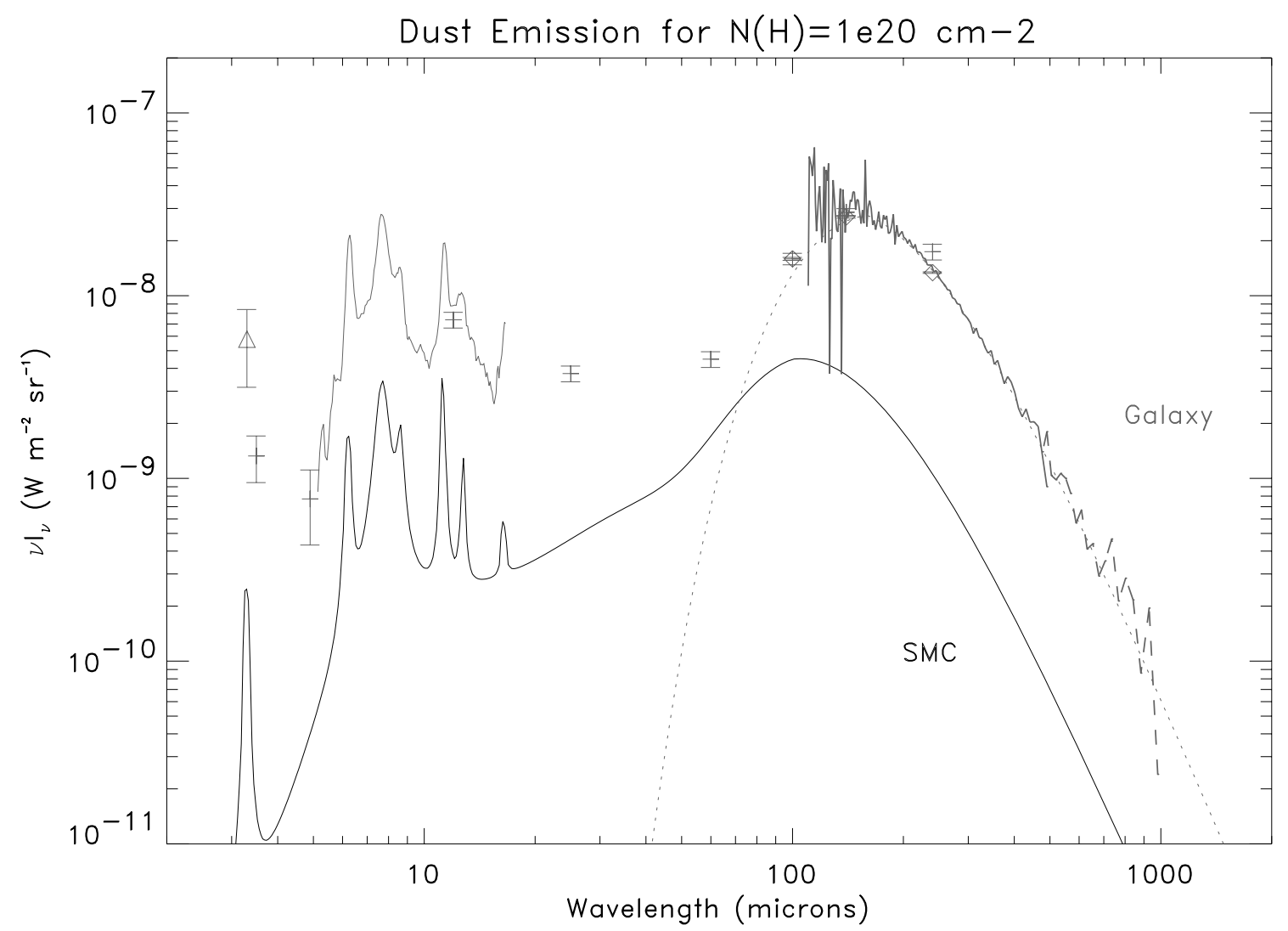

Fig. 9. Infrared spectral energy distribution of dust in the diffuse medium for the SMC and the Solar Neighborhood. The brightness corresponds to an interstellar gas column density of $10^{20} \mathrm{~cm}^{-2}$. The Galactic spectrum (in grey) is composed in the far-IR by COBE/FIRAS data from Boulanger et al. (1996a) (with the corresponding spectrum from grains in thermal equilibrium indicated by the dotted line), and at shorter wavelengths by the COBE/DIRBE (Bernard et al. 1994) and Arome balloon data (triangle at $3.3 \mu \mathrm{m}$ ) (Giard et al. 1994). An ISOCAM spectrum (Boulanger et al. 1996b) scaled to the $12 \mu \mathrm{m}$ brightness is also shown. The SMC spectrum (in black) is the best fit obtained with the Desert et al. model, same as in Fig. 8 .

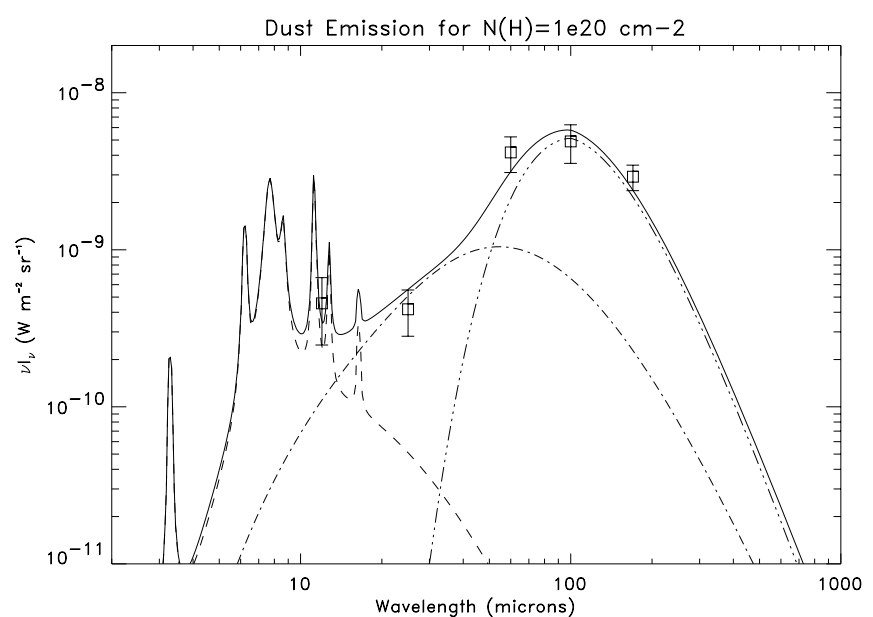

Fig. 10. Dust infrared emission spectrum fit obtained with the Desert et al. model to adjust the $60 \mu \mathrm{m}$ excess. The IRAS and ISOPHOT data are represented by squares. This spectrum is obtained with a single ISRF scaled by $\chi=10$ and reduced dust grain mass abundances for PAHs (dashed line), very small grains (mixed line) and big grains (dotdot-dot-dashed line).

in the diffuse SMC ISM is much lower than the solar neighbourhood value scaled by the difference in metallicity.
The observed $60 \mu \mathrm{m}$ excess could also be due to a change in the grain size distribution (Galliano et al. 2003). We assumed the Galactic grain size distribution to apply in the SMC. However, by changing the relative mass contributions of the different grain components, we enlarge the discontinuities in the size distribution between the three components of the model. It is necessary to further increase these discontinuities to account for the $60 \mu \mathrm{m}$ excess.

Further investigations are needed to characterize the SMC ISRF and especially the $60 \mu \mathrm{m}$ excess. The intensity and spectral shape of the ISRF are likely to be different than the Galactic one.

\section{Discussion}

Studying the gas to dust correlation in the diffuse medium of the SMC (see Sect. 5.2), we found a GDR in mass around 30 times greater than in the solar neighborhood, larger by a factor of 3 than the difference in metallicity. This result confirms the value found by Stanimirovic et al. (2000) in a similar study. This lower depletion of heavy elements in dust was independently reported by Welty et al. (2001), who found $\mathrm{Mg}$ and $\mathrm{Si}$ to be essentially undepleted in the SMC. It was also reported for a sample of dwarf irregulars by Lisenfeld \& Ferrara (1998). 
Our result in the SMC fits with the relation that they observed between GDR and metallicity.

An interpretation of this relation has been proposed by Hirashita et al. (2002). With a chemical evolution model, they illustrated the dependence of the GDR on the star formation history. Stars generate dust but also contribute to its destruction by supernova shocks. Hirashita et al. (2002) propose a scenario in which an intermittent star formation history leads to a time variable GDR. At some epochs of the galaxy evolution, the formation of dust grains is less efficient than the destruction process by supernova shocks, so that the GDR no longer scales with the metallicity. This model could be applied to the SMC with its specific star formation history (Harris \& Zaritsky 2003). This model is also necessary to account for a possible difference in the GDR between the diffuse and the denser medium.

Extinction studies lead to a significantly smaller value of the GDR, closer to that expected from the Galactic value for a linear dependence with metallicity. Bouchet et al. (1985) used photometric observations of O-B stars in the SMC to derive their extinction curves. They found a GDR value that is about 8 times the Galactic value, which is consistent with the difference in metallicity. More recently, Tumlinson et al. (2002) measured $\frac{N\left(\mathrm{H}_{1}\right)+N\left(\mathrm{H}_{2}\right)}{E(B-V)}$ for a sample of stars observed with FUSE and found a mean value 16 times lower than the Solar neighborhood value. This is closer but still smaller by a factor 2 than our estimate. The difference may be due to the fact that extinction studies are based on lines of sights with high hydrogen column density where a dense interstellar medium is likely to be present. Most of the stars are in the optical bar and/or in star formation regions associated with molecular clouds. The difference in the GDRs inferred in our work and extinction studies might thus point to a dependence on the environment: a decrease of the ratio from the diffuse ISM to star forming regions that might arise from the condensation of heavy elements on dust grains in dense gas and their destruction by supernova shocks in the diffuse ISM. Stanimirovic et al. (2000) have also noted this possibility for the SMC, but it still requires confirmation by further studies.

\section{Summary and conclusions}

This study was made feasible by the availability of 10 ISOPHOT observations at $170 \mu \mathrm{m}$, covering most of the SMC, in the ISO Data Archive. After reduction, mapping, and Galactic foreground removal, the ISOPHOT data were compared with IRAS maps and an ATCA/Parkes combined H I column density map to assess the properties of the dust in the diffuse medium of this low-metallicity galaxy.

For the dust in the diffuse medium of the SMC, we found a reference equilibrium temperature of $22 \pm 2 \mathrm{~K}$ and a reference dust emissivity of $3.4 \times 10^{-27} \pm 1.5 \times 10^{-27}\left(\frac{\lambda}{250 \mu \mathrm{m}}\right) \mathrm{cm}^{2}$ per hydrogen atom. This leads to a gas-to-dust ratio 30 times greater than in the Galaxy. This high value reflects the SMC low metallicity, but also requires a lower depletion of dust elements than in the Galaxy. This shows that a simple linear dependence between the GDR and the metallicity does not apply for the diffuse medium of the SMC.
The spectral energy distribution is modeled with the Desert et al. (1990) model. The best fit obtained shows a decrease for PAHs and very small grains similar to that for big grains. However, a $60 \mu \mathrm{m}$ excess remains that cannot be accounted for with simple assumptions. Further constraints, in particular on the ISRF, are needed to understand it.

Our GDR estimate for the diffuse medium of the SMC is a factor 2 to 3 higher than that derived from extinction studies towards bright UV stars with high foreground hydrogen column densities. This difference supports the notion of variations in the GDR, from diffuse regions where grains are more frequently destroyed by supernovae, to denser and more quiescent regions where grains re-accrete heavy elements.

Acknowledgements. We thank the referee for usefull comments which improved the quality of this paper. We acknowledge S. Stanimirovic for providing us with the ATCA/Parkes combined $\mathrm{H}$ I data and her help in using it, and C. Brüns for providing us with the Galactic foreground Hi map. This study has been supported by the French national program PCMI (Physique et Chimie du Milieu Interstellaire, CNRS).

\section{References}

Aguirre, J. E., Bezaire, J. J., Cheng, E. S., et al. 2003, ApJ, 596, 273 Aumann, H. H., Fowler, J. W., \& Melnyk, M. 1990, AJ, 99, 1674

Azzopardi, M., Lequeux, J., \& Maeder, A. 1988, A\&A, 189, 34

Bernard, J. P., Boulanger, F., Desert, F. X., et al. 1994, A\&A, 291, L5

Bontekoe, T. R., Koper, E., \& Kester, D. J. M. 1994, A\&A, 284, 1037

Bouchet, P., Lequeux, J., Maurice, E., Prevot, L., \& Prevot-Burnichon, M. L. 1985, A\&A, 149, 330

Boulanger, F., Abergel, A., Bernard, J.-P., et al. 1996a, A\&A, 312, 256

Boulanger, F., Reach, W. T., Abergel, A., et al. 1996b, A\&A, 315, L325

Brüns, C., Kerp, J., \& Staveley-Smith, L. 2000, in Mapping the Hidden Universe: The Universe behind the Milky Way - The Universe in HI, ASP Conf. Ser., 218, 349

Desert, F.-X., Boulanger, F., \& Puget, J. L. 1990, A\&A, 237, 215

Dufour, R. J. 1984, in Structure and Evolution of the Magellanic Clouds, IAU Symp., 108, 353

Dwek, E. 1998, ApJ, 501, 643

Gabriel, C., Acosta-Puido, J., \& Heinrichsen, I. 1998, ASP Conf. Ser., 145,165

Gabriel, C., Acosta-Puido, J., Kinkel, U., Klaas, U., \& Schulz, B. 1997, ASP Conf. Ser., 125, 108

Galliano, F., Madden, S. C., Jones, A. P., et al. 2003, A\&A, 407, 159

Giard, M., Lamarre, J. M., Pajot, F., \& Serra, G. 1994, A\&A, 286, 203

Harris, J., \& Zaritsky, D. 2003 [arXiv: astro-ph0312100]

Hirashita, H. 1999, ApJ, 522, 220

Hirashita, H., Tajiri, Y. Y., \& Kamaya, H. 2002, A\&A, 388, 439

Issa, M. R., MacLaren, I., \& Wolfendale, A. W. 1990, A\&A, 236, 237

Kerton, C. R., \& Martin, P. G. 2001, PASP, 113, 872

Kessler, M. F., Steinz, J. A., Anderegg, M. E., et al. 1996, A\&A, 315, L27

Lagache, G., \& Dole, H. 2001, A\&A, 372, 702

Lagache, G., Abergel, A., Boulanger, F., \& Puget, J.-L. 1998, A\&A, 333, 709

Lagache, G., Abergel, A., Boulanger, F., Désert, F. X., \& Puget, J.-L. 1999, A\&A, 344, 322

Laureijs, R. J., Klaas, U., Richards, P. J., Schulz, B., \& Abraham, P. 2003, The ISO Handbook, Volume IV - PHT - The Imaging PhotoPolarimeter

Lemke, D., Klaas, U., Abolins, J., et al. 1996, A\&A, 315, L64 
Lequeux, J. 1979, A\&A, 71, 1

Li, A., \& Draine, B. T. 2002, ApJ, 576, 762

Lisenfeld, U., \& Ferrara, A. 1998, ApJ, 496, 145

Lisenfeld, U., \& Ferrara, A. 1997, Dwarf Galaxies: Probes for Galaxy

Formation and Evolution, Joint Discussion 2, 21 August, Kyoto, Japan, 2

Mathis, J. S., Mezger, P. G., \& Panagia, N. 1983, A\&A, 128, 212

Pagani, L., Lagache, G., Bacmann, A., et al. 2003, A\&A, 406, L59

Rubio, M., Lequeux, J., \& Boulanger, F. 1993a, A\&A, 271, 9

Rubio, M., Lequeux, J., Boulanger, F., et al. 1993b, A\&A, 271, 1

Sauvage, M., \& Vigroux, L. 1991, in The Magellanic Clouds, IAU Symp., 148, 407

Sauvage, M., Vigroux, L., \& Thuan, T. X. 1990, A\&A, 237, 296

Schwering, P. B. W., \& Israel, F. P. 1989, A\&AS, 79, 79

Sofia, U. J., \& Meyer, D. M. 2001a, ApJ, 558, L147

Sofia, U. J., \& Meyer, D. M. 2001b, ApJ, 554, L221

Stanimirovic, S., Staveley-Smith, L., Dickey, J. M., Sault, R. J., \& Snowden, S. L. 1999, MNRAS, 302, 417
Stanimirovic, S., Staveley-Smith, L., van der Hulst, J. M., et al. 2000, MNRAS, 315, 791

Staveley-Smith, L., Kim, S., Putman, M., \& Stanimirović, S. 1998, Rev. of Modern Astronomy, 11, 117

Tumlinson, J., Shull, J. M., Rachford, B. L., et al. 2002, ApJ, 566, 857 Vangioni-Flam, E., Lequeux, J., Maucherat-Joubert, M., \& Rocca-Volmerange, B. 1980, A\&A, 90, 73

Welty, D. E., Lauroesch, J. T., Blades, J. C., Hobbs, L. M., \& York, D. G. 2001, ApJ, 554, L75

Westerlund, B. E. 1991, in The Magellanic Clouds, IAU Symp., 148, 15

Wheelock, S. L., Gautier, T. N., Chillemi, J., et al. 1994, NASA STI/Recon Technical Report N, 95, 22539

Wilke, K., Klaas, U., Lemke, D., et al. 2004, A\&A, 414, 69

Wilke, K., Stickel, M., Haas, M., et al. 2003, A\&A, 401, 873 